\title{
Research on Heat Transfer Dynamic Characteristics of Composite Layer Based on Laplace Transform
}

\author{
Chunyu $\mathrm{Xu}^{1,4}$, Junhua Lin ${ }^{2,4}$, Wenhao Liu ${ }^{2}$ and Yuanbiao Zhang ${ }^{3,4}$ \\ ${ }^{1}$ College of Electrical and Information Engineering, Jinan University, Zhuhai, \\ 519070, China; supercrazycyu@ gmail.com \\ ${ }^{2}$ International Business School, Jinan University, Zhuhai, 519070, \\ China;13680324199@163.com(J.L.); $1724633808 @$ qq.com(W.L.) \\ ${ }^{3}$ Packaging Engineering Institute, Zhuhai Campus, Jinan University, Zhuhai, \\ 519070, China \\ ${ }^{4}$ Innovation Practice Base of Mathematical Modeling, Jinan University,Zhuhai, \\ 519070, China \\ * Correspondence: 13680324199@163.com; Tel.: +86-13680324199
}

\begin{abstract}
This paper predict and effectively control the temperature distribution of the steady-state and transient states of anisotropic four-layer composite materials online, knowing the density, specific heat, heat conductivity and thickness of the composite materials. Based on the transfer function, a mathematical model was established to study the dynamic characteristics of heat transfer of the composite materials. First of all, the Fourier heat transfer law was used to establish a one-dimensional Fourier heat conduction differential equation for each composite layer, and the Laplace transformation was carried out to obtain the system function. Then the approximate second-order transfer function of the system was obtained by Taylor expansion, and the Laplace inverse transformation was carried out to obtain the transfer function of the whole system in the time domain. Finally, the accuracy of the simplified analytical solutions of the first, second and third order approximate transfer functions was compared with computer simulation. The results showed that the second order approximate transfer functions can describe the dynamic process of heat transfer better than others. The research on the dynamic characteristics of heat transfer in the composite layer and the dynamic model of heat transfer in composite layer proposed in this paper have a reference value for practical engineering application. It can effectively predict the temperature distribution of composite layer material and reduce the cost of experimental measurement of heat transfer performance of materials.
\end{abstract}

Keywords: Flourier law of heat transfer; Temperature distribution; Laplace transform

\section{Introduction}

Heat transfer is the most common natural phenomenon, and almost all the engineering fields will encounter problems of heat transfer under specific conditions, especially the heat transfer problem of heterogeneous materials, especially for multi-layer heterogeneous materials. The engineering application research of heat 
transfer dynamic characteristics has also spanned the traditional energy power and energy-saving areas of the process. It has been involved in the preparation and processing of materials, the development of space technology, the temperature control of information devices, biotechnology, medicine, environmental purification, and ecological maintenance, as well as the modernization of agricultural engineering and armament. However, most of the practical applications use temperature measuring instruments to study the heat transfer performance and characteristics by simulating the temperature measurement method. It can be seen that the quantitative study of the dynamic characteristics of heat transfer has a very important reference value for the application of practical engineering [1].

In the field of heat transfer, the earliest research was that French physicist Biot (1804) put forward the law of heat conduction through the flat-wall heat conduction experiment, which is the differential form of the later Fourier law. Jean Baptiste Joseph Fourier (1822), a French mathematician and physicist, discovered the basic law of heat conduction and promoted further research in the field of heat the transfer in academia. Currently, in the research field of the dynamic heat transfer process, most scholars adopt transfer function method and state space method. Wang Jicheng et al. (1991) gave the mathematical model of the state space method of heat transfer, which used to control the heat transfer process [2]. Chen Zhijiu et al. (1998) used the transfer function method to give the mathematical model of multi-layer wall heat transfer [3]. The results obtained by these methods are in the form of a transcendental equation or infinite series. Wang Zenggang (2006) used transfer function method to study the dynamic characteristics of a heat transfer process of the two-layer composite plate and obtained the transfer function of heat transfer of composite plate [4] [5]. Mahanthesh, B.(2016) use the Laplace to solve the problem of conjugate effects of heat and mass transfer[6]. Based on the transfer function matrix, Yang Minghan(2016) developed a dynamic model to study the temperature through the Laplace transform of a one-dimensional system LFR control characteristics[7]. Hussanan Abid (2017) obtained the thermal conductivity of nanofluids with the Laplace transform method[8]. Ai Zhiyong(2018) proposed a precise solution for coupled thermohydrodynamics by constructing a standard difference matrix equation and using Laplace-Fourier transform to derive standard differential equations[9].In this paper, the Laplace transform method is used to study the dynamic characteristics of heat transfer in four-layer composite materials. Laplace transform is a classical time-frequency domain transform method, and its transform domain is complex frequency domain. The Laplace transform method is one of the indispensable methods to study continuous-time systems, which makes it widely used in many scientific research fields such as electricity, mechanics and so on, and has been classified into engineering technology research.

Through the Laplace transform, the differential and integral operations of the system in the time domain can be converted into the multiplication and division operations of the $\mathrm{S}$ domain, and the differential and integral equations are transformed into algebraic equations, which simplify the solving steps and reduce the calculation amount greatly. Using Laplace transform, the matrix differential equation with initial value can also be transformed into algebraic equation, and then solved by inverse Laplace transform. This research use the advantage of Laplace transform to solve the problem of thick laminated plates under complex boundary conditions [10] [11] [12].

The structure of this paper is as follows: The first part is the introduction, the second part is an introduction to the heat transfer model, the Fourier differential equation is established for each layer of composite material and the Laplace transform is performed to solve the system function. The third part is the application of the model, 
using step function to test the accuracy of the model. The fourth part is the conclusion of the model.

\section{Establishment of heat transfer model}

\subsection{Model assumptions}

As shown in Figure 1, the seamlessly bonded composite materials that make up the heat transfer system are processed by layering and boundary method. The following assumptions are made:

(1) Each layer of material is a composite plane with uniform thickness and infinite area, and the temperature on both sides of the steel is uniform;

(2) The joint faces between different material layers are tight, and there is no contact thermal resistance on the joint faces of different materials;

(3) The various properties of the single-layer material, such as density, specific heat capacity, thermal conductivity, etc., are uniform, so that the temperature on the same layer is the same;

(4) The thickness of each layer is uniform and thin;

(5) There is no heat source or sink in the material, that is, there is no increase or loss during the heat transfer.
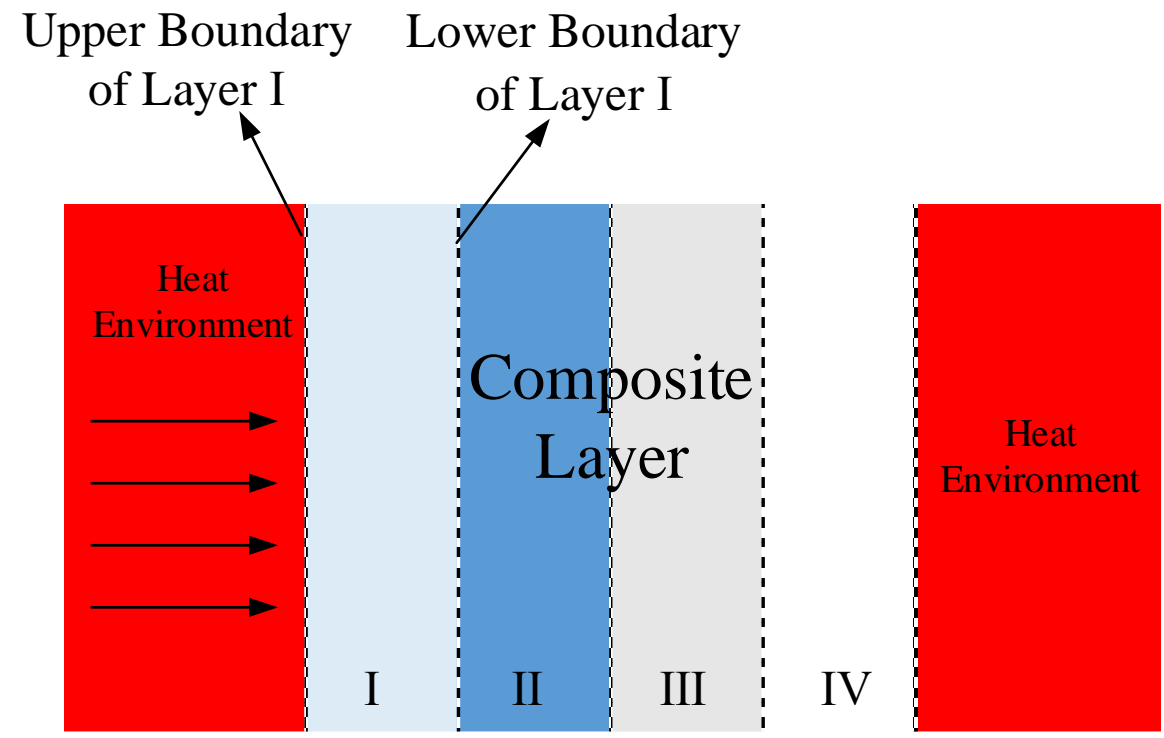

Figure 1. Heat transfer diagram

Based on the assumptions above, the heat transfer of the composite layer can be regarded as the one-dimensional heat transfer, and a one-dimensional heat conduction Fourier differential equation can be established:

$$
\begin{gathered}
\frac{d^{2} t(x, \tau)}{d x^{2}}-\frac{1}{a} \cdot \frac{d t(x, \tau)}{d \tau}=0 \\
a=\frac{\lambda}{\rho c}
\end{gathered}
$$

In the equation, $t(x, \tau)$ indicates that the temperature is a function of time $t$ and material thickness of $x, \lambda 、 \rho 、 c$ represent the thermal conductivity, density and specific heat capacity of the material, and $a$ is indicated the thermal conductivity of the object. 
The heat conduction Fourier differential equation represents the relationship of temperature with time at a certain thickness. Apply Laplace transform to (1), let $T(x, s)=\mathrm{L}[t(x, \tau)]$, there are:

$$
\mathrm{L}\left[\frac{d^{2} t(x, \tau)}{d x^{2}}\right]-\frac{1}{a} L\left[\frac{d t(x, \tau)}{d t}\right]=0
$$

That is:

$$
\frac{d^{2} T(x, s)}{d x^{2}}-\frac{1}{a} \cdot s \cdot T(x, s)=0
$$

Where $T(x, s)$ is the Laplace transform of $t(x, s)$, and the general solution of (4) is:

$$
\begin{gathered}
T(x, s)=A e^{q x}+B e^{-q x} \\
q=\sqrt{s / a}
\end{gathered}
$$

Among them, $A$ and $B$ are the coefficients that need to be solved. $A, B$ are independent of $x$ and can be related to $S$.

Based on the Fourier heat transfer law, the boundary temperature of each layer of material is analyzed separately. Each layer has two boundaries which are defined as the upper and lower boundaries. The temperature distribution model of the heat transfer system can be solved by identifying the temperature on the joint surfaces of the two adjacent layers of the composite plate.

\subsection{Boundary temperature analysis of the first layer of material}

In the first layer, the thickness of the layer material is $\delta_{1}$, and the general solution of the heat conduction Fourier differential equation is:

$$
T_{\delta_{1}}(x, s)=A_{\delta_{1}} \cdot e^{q_{\delta_{1}} x}+B_{\delta_{1}} \cdot e^{-q_{\delta_{1}} x}
$$

Where $x \in\left[0, \delta_{1}\right]$, and $q_{\delta_{1}}=\sqrt{\frac{s}{a_{1}}}$.

When the entire heat transfer system is in the final thermal equilibrium state, regardless of the loss of heat transfer, the upper boundary temperature of the first layer of material should be infinitely close to the constant temperature $t_{0}$, that is:

$$
\begin{gathered}
t_{\delta_{1}}(0, \tau)=t_{0} \\
\left.\frac{d t_{\delta_{1}}(x, \tau)}{d x}\right|_{x=\delta_{1}}=\frac{1}{\lambda_{\delta_{1}}} v_{1}\left(\delta_{1}, \tau\right)
\end{gathered}
$$

Where $\delta_{1}$ is the thickness of the material of the first layer, $v_{1}\left(s_{1}, \tau\right)$ is the heat flux density of the material of the first layer, which is a function to be determined, $\lambda_{\delta 1}$ is the thermal conductivity of the layer, Laplace transform is applied to the upper and lower boundaries [3-7]:

$$
T_{\delta_{1}}(0, s)=\frac{t_{0}}{s}
$$




$$
\mathrm{L}\left\{\left.\frac{d t_{\delta 1}(x, \tau)}{d x}\right|_{x=\delta_{1}}\right\}=\frac{1}{\lambda_{\delta_{1}}} V_{1}\left(\delta_{1}, s\right)
$$

Where $V_{1}\left(\delta_{1}, s\right)$ is the Laplace transform of $v_{1}\left(s_{1}, \tau\right), T_{\delta_{1}}(0, s)$ is the Laplace transform of $t_{\delta_{1}}(x, \tau)$, L is Pull transformation operator.

From equations (5) and (9), we can get that:

$$
T_{\delta_{1}}(0, s)=A_{\delta_{1}}+B_{\delta_{1}}=\frac{t_{0}}{s}
$$

Since $\mathrm{L}\left\{\left.\frac{d t_{\delta_{1}}(x, \tau)}{d x}\right|_{x=\delta_{1}}\right\}=\left.\frac{d T_{\delta_{1}}(x, s)}{d x}\right|_{x=\delta_{1}}$, Therefore, the substitution of known quantities for formula (10) is available:

$$
A_{\delta_{1}} \cdot q_{\delta_{1}} \cdot e^{q_{\delta_{1}} \delta_{1}}-B_{\delta_{1}} \cdot q_{\delta_{1}} \cdot e^{-q_{\delta_{1}} \delta_{1}}=\frac{1}{\lambda_{\delta_{1}}} \cdot V_{1}\left(\delta_{1}, s\right)
$$

Thus, the simultaneous formulas (11) and (12) can be solved as follows:

$$
\left\{\begin{array}{c}
A_{\delta_{1}}=\frac{\frac{1}{\lambda_{\delta_{1}}} V_{1}\left(\delta_{1}, s\right)+q_{\delta_{1}} \cdot e^{-q_{\delta_{1}} \delta_{1}} \cdot \frac{t_{0}}{s}}{2 q_{\delta_{1}} \cosh \left(q_{\delta_{1}} \delta_{1}\right)} \\
B_{\delta_{1}}=\frac{\frac{t_{0}}{s} \cdot q_{\delta_{1}} \cdot e^{q_{\delta_{1}} \delta_{1}}-\frac{1}{\lambda_{\delta_{1}}} V_{1}\left(\delta_{1}, s\right)}{2 q_{\delta_{1}} \cosh \left(q_{\delta_{1}} \delta_{1}\right)}
\end{array}\right.
$$

By substituting equation (13) into equation (5) and using the definition of hyperbola, the following results are obtained:

$$
T_{\delta_{1}}(x, s)=\frac{\frac{1}{\lambda_{\delta_{1}}} V_{1}\left(\delta_{1}, s\right) \sinh \left(q_{\delta_{1}} x\right)+\frac{t_{0}}{s} q_{\delta_{1}} \cosh \left[q_{\delta_{1}}\left(x-\delta_{1}\right)\right]}{q_{\delta_{1}} \cosh \left(q_{\delta_{1}} \delta_{1}\right)}
$$

Where $x \in\left[0, \delta_{1}\right]$ and $q_{\delta_{1}}=\sqrt{\frac{s}{a_{1}}}$.

When $x=\delta_{1}$, the temperature of the lower boundary of the material of the first layer is obtained:

$$
T_{\delta_{1}}(x, s)=\frac{\frac{1}{\lambda_{\delta_{1}}} V_{1}\left(\delta_{1}, s\right) \sinh \left(q_{\delta_{1}} \delta_{1}\right)+\frac{t_{0}}{s} q_{\delta_{1}}}{q_{\delta_{1}} \cosh \left(q_{\delta_{1}} \delta_{1}\right)}
$$

\subsection{Boundary temperature analysis of the second layer of material}

In this layer, it is recorded that the thickness of the layer material is $\delta_{2}$, and the general solution of the heat conduction Fourier differential equation is as follows: 


$$
T_{\delta_{2}}(x, s)=A_{\delta_{2}} \cdot e^{q_{\delta_{2}}\left(x-\delta_{1}\right)}+B_{\delta_{2}} \cdot e^{-q_{\delta_{2}}\left(x-\delta_{1}\right)}
$$

Where $x \in\left[\delta_{1}, \delta_{1}+\delta_{2}\right]$, and: $q_{\delta_{2}}=\sqrt{\frac{s}{a_{2}}}$.

Since the heat flux density on both sides of the joint surface is the same, the lower boundary of the first layer is the upper boundary of the second layer, the temperature at the joint surface is the same, so the boundary condition can be obtained:

$$
\left\{\begin{array}{c}
\left.\frac{d t_{\delta_{2}}(x, \tau)}{d x}\right|_{x=\delta 1}=\frac{1}{\lambda_{\delta_{2}}} v_{1}\left(\delta_{1}, \tau\right) \\
\left.\frac{d t_{\delta_{2}}(x, \tau)}{d x}\right|_{x=\delta 1+\delta_{2}}=\frac{1}{\lambda_{\delta_{2}}} v_{2}\left(\delta_{1}+\delta_{2}, \tau\right)
\end{array}\right.
$$

Performing Laplacian transformation on equation (17) yields:

$$
\left\{\begin{array}{c}
\mathrm{L}\left\{\left.\frac{d t_{\delta_{2}}(x, \tau)}{d x}\right|_{x=\delta 1}\right\}=\frac{1}{\lambda_{\delta_{2}}} V_{1}\left(\delta_{1}, s\right) \\
\mathrm{L}\left\{\left.\frac{d t_{\delta_{2}}(x, \tau)}{d x}\right|_{x=\delta_{1}+\delta_{2}}\right\}=\frac{1}{\lambda_{\delta_{2}}} V_{2}\left(\delta_{1}+\delta_{2}, s\right)
\end{array}\right.
$$

Where $V_{1}\left(\delta_{1}, s\right)$ is Laplace transform of $v_{1}\left(s_{1}, \tau\right), V_{2}\left(\delta_{1}+\delta_{2}, s\right)$ is Laplace transform of $v_{2}\left(\delta_{1}+\delta_{2}, \tau\right)$, and L is Pull transformation operator.

Since $L\left\{\left.\frac{d t_{\delta_{2}}(x, \tau)}{d x}\right|_{x=\delta_{1}}\right\}=\left.\frac{d T_{\delta_{2}}(x, s)}{d x}\right|_{x=\delta_{1}}, \mathrm{~L}\left\{\left.\frac{d t_{\delta_{2}}(x, \tau)}{d x}\right|_{x=\delta_{1}+\delta_{2}}\right\}=\left.\frac{d T_{\delta_{2}}(x, s)}{d x}\right|_{x=\delta_{1}+\delta_{2}}$ so the formula for the general solution of the formula (18) is:

$$
\left\{\begin{array}{c}
A_{\delta_{2}} q_{\delta_{2}}-B_{\delta_{2}} q_{\delta_{2}}=\frac{1}{\lambda_{\delta_{2}}} V_{1}\left(\delta_{1}, s\right) \\
A_{\delta_{2}} q_{\delta_{2}} e^{q_{\delta_{2}} \delta_{2}}-B_{\delta_{2}} \cdot q_{\delta_{2}} \cdot e^{-q_{\delta_{2}} \delta_{2}}=\frac{1}{\lambda_{\delta_{2}}} V_{2}\left(\delta_{1}+\delta_{2}, s\right)
\end{array}\right.
$$

Thus, the equations of equation (19) can be solved to obtain the constant coefficients of the layer of thermally conductive Fourier differential equations:

$$
\left\{\begin{array}{c}
A_{\delta_{2}}=\frac{V_{2}\left(\delta_{1}+\delta_{2}, s\right)-V_{1}\left(\delta_{1}, s\right) e^{-q_{\delta_{2}} \delta_{2}}}{2 \lambda_{\delta_{2}} q_{\delta_{2}} \sinh \left(q_{\delta_{2}} \delta_{2}\right)} \\
B_{\delta_{2}}=\frac{V_{2}\left(\delta_{1}+\delta_{2}, s\right)-V_{1}\left(\delta_{1}, s\right) e^{q_{\delta_{2}} \delta_{2}}}{2 \lambda_{\delta_{2}} q_{\delta_{2}} \sinh \left(q_{\delta_{2}} \delta_{2}\right)}
\end{array}\right.
$$

Substituting equation (20) into general solution (16) yields:

$$
T_{\delta_{2}}(x, s)=\frac{r_{1}-r_{2}}{\lambda_{\delta_{2}} q_{\delta_{2}} \sinh \left(q_{\delta_{2}} \delta_{2}\right)}, x \in\left[\delta_{1}, \delta_{1}+\delta_{2}\right]
$$


among them,

$$
\left\{\begin{array}{l}
r_{1}=V_{2}\left(\delta_{1}+\delta_{2}, s\right) \cosh \left[q_{\delta_{2}}\left(x-\delta_{1}\right)\right] \\
r_{2}=V_{1}\left(\delta_{1}, s\right) \cosh \left[q_{\delta_{2}}\left(\delta_{1}+\delta_{2}-x\right)\right]
\end{array}\right.
$$

When $x=\delta_{1}$, the temperature at the upper boundary of the second layer is obtained as follows:

$$
T_{\delta_{2}}\left(\delta_{1}, s\right)=\frac{V_{2}\left(\delta_{1}+\delta_{2}, s\right)-V_{1}\left(\delta_{1}, s\right) \cosh \left(q_{\delta_{2}} \delta_{2}\right)}{\lambda_{\delta_{2}} q_{\delta_{2}} \sinh \left(q_{\delta_{2}} \delta_{2}\right)}
$$

When $x=\delta_{1}+\delta_{2}$, the temperature at the lower boundary of the second layer is obtained as follows:

$$
T_{\delta_{2}}\left(\delta_{1}+\delta_{2}, s\right)=\frac{V_{2}\left(\delta_{1}+\delta_{2}, s\right) \cosh \left(q_{\delta_{2}} \delta_{2}\right)-V_{1}\left(\delta_{1}, s\right)}{\lambda_{\delta_{2}} q_{\delta_{2}} \sinh \left(q_{\delta_{2}} \delta_{2}\right)}
$$

\subsection{Boundary temperature analysis of the third layer of material}

In this layer, it is recorded that the thickness of the layer material is $\delta_{3}$, and the general solution of the heat conduction Fourier differential equation is as follows:

$$
T_{\delta_{3}}(x, s)=A_{\delta_{3}} \cdot e^{q_{\delta_{3}}\left(x-\delta_{1}-\delta_{2}\right)}+B_{\delta_{3}} \cdot e^{-q_{\delta_{3}}\left(x-\delta_{1}-\delta_{2}\right)}
$$

Where $x \in\left[\delta_{1}+\delta_{2}, \delta_{1}+\delta_{2}+\delta_{3}\right]$, and: $q_{\delta_{3}}=\sqrt{\frac{s}{a_{3}}}$.

The differential equation of the upper and lower boundaries of the third layer material is as follows:

$$
\left\{\begin{array}{c}
\left.\frac{d t_{\delta_{3}}(x, \tau)}{d x}\right|_{x=\delta_{1}+\delta_{2}}=\frac{1}{\lambda_{\delta_{3}}} v_{2}\left(\delta_{1}+\delta_{2}, \tau\right) \\
\left.\frac{d t_{\delta 2}(x, \tau)}{d x}\right|_{x=\delta_{1}+\delta_{2}+\delta_{3}}=\frac{1}{\lambda_{\delta_{3}}} v_{3}\left(\delta_{1}+\delta_{2}+\delta_{3}, \tau\right)
\end{array}\right.
$$

Performing Laplacian transformation on equation (26) yields:

$$
\left\{\begin{array}{c}
\mathrm{L}\left\{\left.\frac{d t_{\delta_{3}}(x, \tau)}{d x}\right|_{x=\delta_{1}+\delta_{2}}\right\}=\frac{1}{\lambda_{\delta_{3}}} V_{2}\left(\delta_{1}+\delta_{2}, s\right) \\
\mathrm{L}\left\{\left.\frac{d t_{\delta_{3}}(x, \tau)}{d x}\right|_{x=\delta_{1}+\delta_{2}+\delta_{3}}\right\}=\frac{1}{\lambda_{\delta_{3}}} V_{3}\left(\delta_{1}+\delta_{2}+\delta_{3}, s\right)
\end{array}\right.
$$

Where $V_{2}\left(\delta_{1}+\delta_{2}, s\right)$ is Laplace transform of $v_{2}\left(\delta_{1}+\delta_{2}, \tau\right), V_{3}\left(\delta_{1}+\delta_{2}+\right.$ $\left.\delta_{3}, s\right)$ is Laplace transform of $v_{3}\left(\delta_{1}+\delta_{2}+\delta_{3}, \tau\right)$, and $\mathrm{L}$ is Pull transformation operator.

The formula for the general solution of the formula (27) is:

$$
\left\{\begin{array}{c}
A_{\delta_{3}} q_{\delta_{3}}-B_{\delta_{3}} q_{\delta_{3}}=\frac{1}{\lambda_{\delta_{3}}} V_{2}\left(\delta_{1}+\delta_{2}, s\right) \\
A_{\delta_{3}} q_{\delta_{3}} e^{q_{\delta_{3}} \delta_{3}}-B_{\delta_{3}} \cdot q_{\delta_{3}} \cdot e^{-q_{\delta_{3}} \delta_{3}}=\frac{1}{\lambda_{\delta_{3}}} V_{3}\left(\delta_{1}+\delta_{2}+\delta_{3}, s\right)
\end{array}\right.
$$


Thus, the equations of equation (28) can be solved to obtain the constant coefficients of the layer of thermally conductive Fourier differential equations:

$$
\left\{\begin{array}{c}
A_{\delta_{3}}=\frac{V_{3}\left(\delta_{1}+\delta_{2}+\delta_{3}, s\right)-V_{2}\left(\delta_{1}+\delta_{2}, s\right) e^{-q_{\delta_{3}} \delta_{3}}}{2 \lambda_{\delta_{3}} q_{\delta_{3}} \sinh \left(q_{\delta_{3}} \delta_{3}\right)} \\
B_{\delta_{3}}=\frac{V_{3}\left(\delta_{1}+\delta_{2}+\delta_{3}, s\right)-V_{2}\left(\delta_{1}+\delta_{2}, s\right) e^{q_{\delta_{3}} \delta_{3}}}{2 \lambda_{\delta_{3}} q_{\delta_{3}} \sinh \left(q_{\delta_{3}} \delta_{3}\right)}
\end{array}\right.
$$

Substituting equation (29) into general solution (25) yields:

$$
\begin{aligned}
T_{\delta_{3}}(x, s)= & \frac{r_{3}-r_{4}}{\lambda_{\delta_{3}} q_{\delta_{3}} \sinh \left(q_{\delta_{3}} \delta_{3}\right)}, x \\
& \in\left[\delta_{1}+\delta_{2}, \delta_{1}+\delta_{2}+\delta_{3}\right]
\end{aligned}
$$

among them,

$$
\left\{\begin{array}{l}
r_{3}=V_{3}\left(\delta_{1}+\delta_{2}+\delta_{3}, s\right) \cosh \left[q_{\delta_{3}}\left(x-\delta_{1}-\delta_{2}\right)\right] \\
r_{4}=V_{2}\left(\delta_{1}+\delta_{2}, s\right) \cosh \left[q_{\delta_{3}}\left(\delta_{1}+\delta_{2}+\delta_{3}-x\right)\right]
\end{array}\right.
$$

When $x=\delta_{1}+\delta_{2}$, the temperature at the upper boundary of the third layer is obtained as follows:

$$
\begin{aligned}
& T_{\delta_{3}}\left(\delta_{1}+\delta_{2}, s\right) \\
& =\frac{V_{3}\left(\delta_{1}+\delta_{2}+\delta_{3}, s\right)-V_{2}\left(\delta_{1}+\delta_{2}, s\right) \cosh \left(q_{\delta_{3}} \delta_{3}\right)}{\lambda_{\delta_{3}} q_{\delta_{3}} \sinh \left(q_{\delta_{3}} \delta_{3}\right)}
\end{aligned}
$$

When $x=\delta_{1}+\delta_{2}+\delta_{3}$, the temperature at the lower boundary of the second layer is obtained as follows:

$$
\begin{aligned}
& T_{\delta_{3}}\left(\delta_{1}+\delta_{2}+\delta_{3}, s\right) \\
& =\frac{V_{3}\left(\delta_{1}+\delta_{2}+\delta_{3}, s\right) \cosh \left(q_{\delta_{3}} \delta_{3}\right)-V_{2}\left(\delta_{1}+\delta_{2}, s\right)}{\lambda_{\delta_{3}} q_{\delta_{3}} \sinh \left(q_{\delta_{3}} \delta_{3}\right)}
\end{aligned}
$$

\subsection{Boundary temperature analysis of the forth layer of material}

In this layer, it is recorded that the thickness of the layer material is $\delta_{4}$, and the general solution of the heat conduction Fourier differential equation is as follows:

$$
T_{\delta_{4}}(x, s)=A_{\delta_{4}} \cdot e^{q_{\delta_{4}}\left(x-\delta_{1}-\delta_{2}-\delta_{3}\right)}+B_{\delta_{4}} e^{-q_{\delta_{4}}\left(x-\delta_{1}-\delta_{2}-\delta_{3}\right)}
$$

Where $\epsilon \in\left[\delta_{1}+\delta_{2}+\delta_{3}, \delta_{1}+\delta_{2}+\delta_{3}+\delta_{4}\right]$, and: $q_{\delta_{4}}=\sqrt{\frac{s}{a_{4}}}$.

The differential equation of the upper and lower boundaries of the forth layer material is as follows:

$$
\left\{\begin{array}{c}
\left.\frac{d t_{\delta 4}(x, \tau)}{d x}\right|_{x=\delta_{1}+\delta_{2}+\delta_{3}}=\frac{1}{\lambda_{4}} v_{3}\left(\delta_{1}+\delta_{2}+\delta_{3}, \tau\right) \\
\left.\frac{d t_{\delta 4}(x, \tau)}{d x}\right|_{x=\delta 1+\delta_{2}+\delta_{3}+\delta_{4}}=t_{1}
\end{array}\right.
$$

Performing Laplacian transformation on equation (35) yields: 


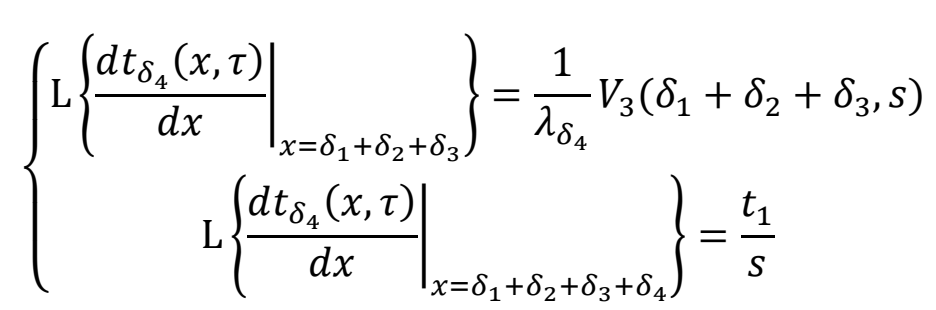

Where $V_{3}\left(\delta_{1}+\delta_{2}+\delta_{3}, s\right)$ is Laplace transform of $v_{3}\left(\delta_{1}+\delta_{2}+\delta_{3}, \tau\right), V_{3}\left(\delta_{1}+\right.$ $\left.\delta_{2}+\delta_{3}, s\right)$ is Laplace transform of $v_{3}\left(\delta_{1}+\delta_{2}+\delta_{3}, \tau\right)$, and L is Pull transformation operator. $\frac{t_{1}}{s}$ is Laplace transform of $t_{1}, t_{1}$ is the lower boundary temperature of the forth layer, which is constant. We can simplify formula (36) and obtain

$$
\left\{\begin{array}{c}
A_{\delta_{4}} q_{\delta_{4}}-B_{\delta_{4}} q_{\delta_{4}}=\frac{1}{\lambda_{\delta_{4}}} V_{3}\left(\delta_{1}+\delta_{2}+\delta_{3}, s\right) \\
A_{\delta_{4}} q_{\delta_{4}} e^{q_{\delta_{4}} \delta_{4}}-B_{\delta_{4}} q_{\delta_{4}} e^{-q_{\delta_{4}} \delta_{4}}=\frac{t_{1}}{s}
\end{array}\right.
$$

Thus, the equations of equation (37) can be solved.

$$
\left\{\begin{array}{c}
A_{\delta_{4}}=\frac{\frac{t_{1}}{s}-\frac{1}{\lambda_{\delta_{4}}} V_{3}\left(\delta_{1}+\delta_{2}+\delta_{3}, s\right) e^{-q_{\delta_{4}} \delta_{4}}}{2 q_{\delta_{4}} \sinh \left(q_{\delta_{4}} \delta_{4}\right)} \\
B_{\delta_{3}}=\frac{\frac{t_{1}}{s}-\frac{1}{\lambda_{\delta_{4}}} V_{3}\left(\delta_{1}+\delta_{2}+\delta_{3}, s\right) e^{q_{\delta_{4}} \delta_{4}}}{2 q_{\delta_{4}} \sinh \left(q_{\delta_{4}} \delta_{4}\right)}
\end{array}\right.
$$

Substituting equation (38) into general solution (34) yields:

$$
T_{\delta_{4}}(x, s)=\frac{r_{5}-r_{6}}{2 q_{\delta_{4}} \sinh \left(q_{\delta_{4}} \delta_{4}\right)}
$$

among them,

$$
\left\{\begin{array}{c}
r_{5}=\frac{t_{1}}{s} \cosh \left[q_{\delta_{4}}\left(x-\delta_{1}-\delta_{2}-\delta_{3}\right)\right] \\
r_{6}=\frac{1}{\lambda_{\delta_{4}}} V_{3}\left(\delta_{1}+\delta_{2}+\delta_{3}, s\right) \cosh \left[q_{\delta_{4}}\left(\delta_{1}+\delta_{2}+\delta_{3}+\delta_{4}-x\right)\right]
\end{array}\right.
$$

Finally, we can obtain the temperature of the upper boundary of the fourth layer.

$$
T_{\delta_{4}}\left(\delta_{1}+\delta_{2}+\delta_{3}, s\right)=\frac{\frac{t_{1}}{s}-\frac{1}{\lambda_{\delta_{4}}} V_{3}\left(\delta_{1}+\delta_{2}+\delta_{3}, s\right) \cosh \left(q_{\delta_{4}} \delta_{4}\right)}{2 q_{\delta_{4}} \sinh \left(q_{\delta_{4}} \delta_{4}\right)}
$$

And the temperature at the lower boundary of the forth layer:

$$
T_{\delta_{4}}\left(\delta_{1}+\delta_{2}+\delta_{3}+\delta_{4}, s\right)=\frac{\frac{t_{1}}{s} \cosh \left(q_{\delta_{4}} \delta_{4}\right)-\frac{1}{\lambda_{\delta_{4}}} V_{3}\left(\delta_{1}+\delta_{2}+\delta_{3}, s\right)}{2 q_{\delta_{4}} \sinh \left(q_{\delta_{4}} \delta_{4}\right)}
$$

\subsection{Solution of the model}

Since the temperature of the joint surfaces and the heat flux density are the same, then we can obtain: 


$$
\left\{\begin{aligned}
T_{\delta_{1}}\left(\delta_{1}, s\right) & =T_{\delta_{2}}\left(\delta_{1}, s\right) \\
T_{\delta_{2}}\left(\delta_{1}+\delta_{2}, s\right) & =T_{\delta_{3}}\left(\delta_{1}+\delta_{2}, s\right) \\
T_{\delta_{3}}\left(\delta_{1}+\delta_{2}+\delta_{3}, s\right) & =T_{\delta_{4}}\left(\delta_{1}+\delta_{2}+\delta_{3}, s\right)
\end{aligned}\right.
$$

The system function is:

$$
G(s)=\frac{\text { Input }}{\text { Output }}=\frac{T_{\delta_{4}}\left(\delta_{1}+\delta_{2}+\delta_{3}+\delta_{4}, s\right)}{t_{0} / s}
$$

When calculating the transfer function of the whole system, the lower boundary of the fourth layer is directly exposed to the hot environment, and there is no increase or loss of heat in the process of transmission, it is assumed that the lower boundary of the forth layer is diabatic, that is:

$$
t_{1}=0
$$

Therefore, the expressions of system functions are obtained by combining the above formulas (1) (45)

$$
G(s)=\frac{1}{\sum_{i=1}^{8} p_{i}}
$$

among them:

$$
\left\{\begin{array}{c}
p_{1}=\frac{q_{\delta_{3}} \lambda_{\delta_{3}} \sinh \left(q_{\delta_{2}} \delta_{2}\right) \sinh \left(q_{\delta_{3}} \delta_{3}\right) \cosh \left(q_{\delta_{1}} \delta_{1}\right) \cosh \left(q_{\delta_{4}} \delta_{4}\right)}{q_{\delta_{2}} \lambda_{\delta_{2}}} \\
p_{2}=\frac{2 q_{\delta_{2}} q_{\delta_{4}} \lambda_{\delta_{2}} \lambda_{\delta_{4}} \sinh \left(q_{\delta_{1}} \delta_{1}\right) \sinh \left(q_{\delta_{2}} \delta_{2}\right) \sinh \left(q_{\delta_{3}} \delta_{3}\right) \sinh \left(q_{\delta_{4}} \delta_{4}\right)}{q_{\delta_{1}} q_{\delta_{3}} \lambda_{\delta_{1}} \lambda_{\delta_{3}}} \\
p_{3}=\frac{q_{\delta_{3}} \lambda_{\delta_{3}} \sinh \left(q_{\delta_{1}} \delta_{1}\right) \sinh \left(q_{\delta_{3}} \delta_{3}\right) \cosh \left(q_{\delta_{2}} \delta_{2}\right) \cosh \left(q_{\delta_{4}} \delta_{4}\right)}{q_{\delta_{1}} \lambda_{\delta_{1}}} \\
p_{4}=\frac{q_{\delta_{2}} \lambda_{\delta_{2}} \sinh \left(q_{\delta_{1}} \delta_{1}\right) \sinh \left(q_{\delta_{2}} \delta_{2}\right) \cosh \left(q_{\delta_{3}} \delta_{3}\right) \cosh \left(q_{\delta_{4}} \delta_{4}\right)}{q_{\delta_{1}} \lambda_{\delta_{1}}} \\
p_{5}=\frac{2 q_{\delta_{4}} \lambda_{\delta_{4}} \cosh \left(q_{\delta_{1}} \delta_{1}\right) \cosh \left(q_{\delta_{2}} \delta_{2}\right) \sinh \left(q_{\delta_{3}} \delta_{3}\right) \sinh \left(q_{\delta_{4}} \delta_{4}\right)}{q_{\delta_{3}} \lambda_{\delta_{3}}} \\
p_{6}=\frac{2 q_{\delta_{4}} \lambda_{\delta_{4}} \sinh \left(q_{\delta_{2}} \delta_{2}\right) \sinh \left(q_{\delta_{4}} \delta_{4}\right) \cosh \left(q_{\delta_{1}} \delta_{1}\right) \cosh \left(q_{\delta_{3}} \delta_{3}\right)}{q_{\delta_{2}} \lambda_{\delta_{2}}} \\
p_{7}=\frac{2 q_{\delta_{4}} \lambda_{\delta_{4}} \sinh \left(q_{\delta_{1}} \delta_{1}\right) \sinh \left(q_{\delta_{4}} \delta_{4}\right) \cosh \left(q_{\delta_{2}} \delta_{2}\right) \cosh \left(q_{\delta_{3}} \delta_{3}\right)}{q_{\delta_{1}} \lambda_{\delta_{1}}} \\
p_{8}=\cosh \left(q_{\delta_{1}} \delta_{1}\right) \cosh \left(q_{\delta_{2}} \delta_{2}\right) \cosh \left(q_{\delta_{3}} \delta_{3}\right) \cosh \left(q_{\delta_{4}} \delta_{4}\right)
\end{array}\right.
$$

Expand the system function, the denominator of equation (46) into a Taylor series, and get the polynomial of $s$ :

$$
G(s)=\frac{1}{\omega_{0}+\omega_{1} s+\omega_{2} s^{2}+\omega_{3} s^{3}+\cdots}
$$

among them:

$$
\begin{gathered}
\omega_{0}=1 \\
\omega_{1}=\frac{\lambda_{\delta_{3}} \delta_{2} \delta_{3}}{a_{3} \lambda_{\delta_{2}}}+\frac{\lambda_{\delta_{3}} \delta_{1} \delta_{3}}{a_{3} \lambda_{\delta_{1}}}+\frac{\lambda_{\delta_{2}} \delta_{1} \delta_{2}}{a_{2} \lambda_{\delta_{1}}}+\sum_{i=1}^{3} \frac{2 \lambda_{\delta_{4}} \delta_{i} \delta_{4}}{a_{4} \lambda_{\delta_{i}}}+\sum_{i=1}^{4} \frac{\delta_{i}{ }^{2}}{2 a_{i}}
\end{gathered}
$$




$$
\omega_{2}=d_{1}+d_{2}+d_{3}+2 d_{4}++2 d_{5}++2 d_{6}+d_{7}+d_{8}+d_{9}
$$

And we can obtain:

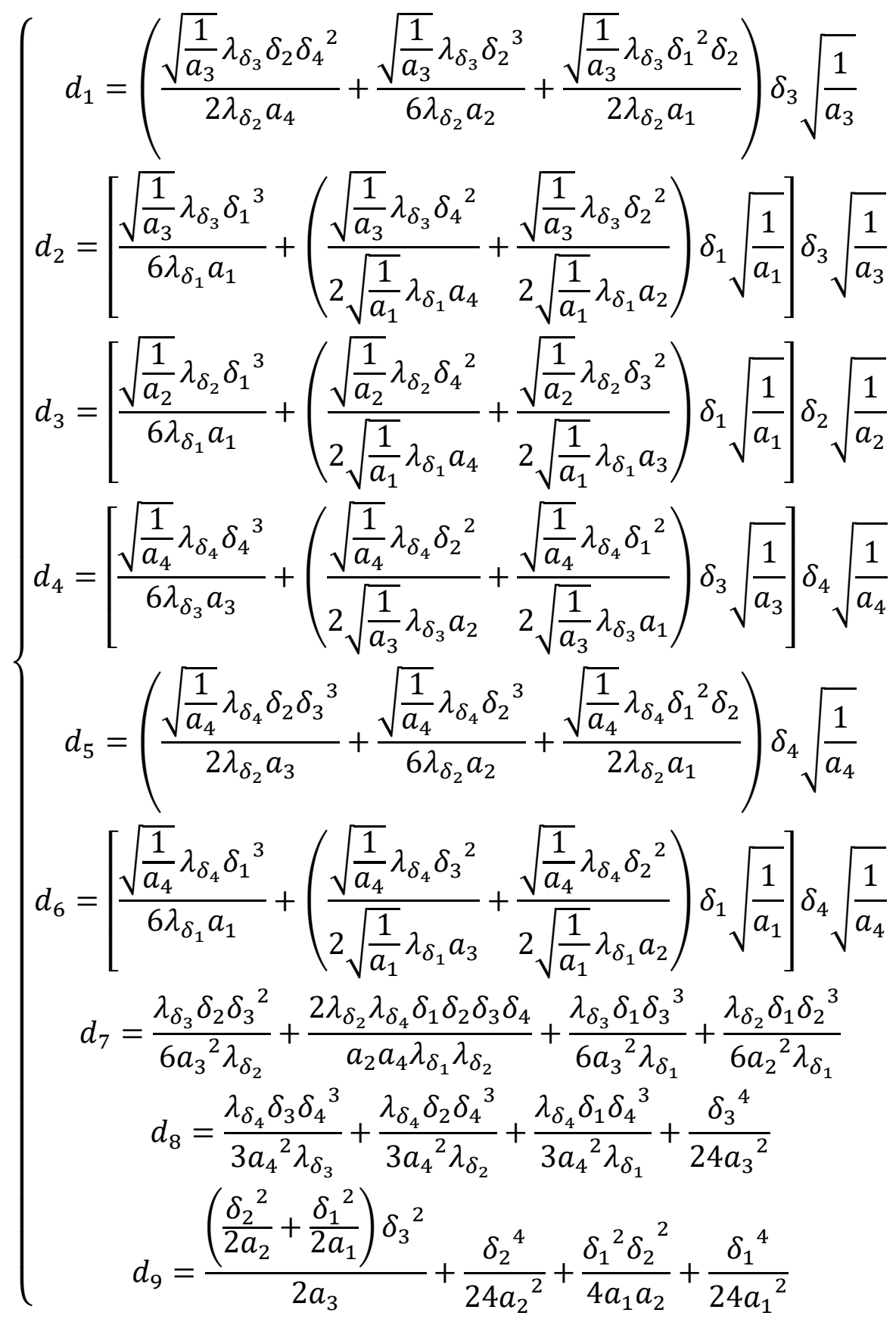

For the system of equations $(52), a_{i}(i=1,2,3,4)$ is the thermal conductivity of the layer $i$

$\delta_{i}(i=1,2,3,4)$ is the thickness of layer $i$ and $\lambda_{\delta_{i}}(i=1,2,3,4)$ is the thermal conductivity of the layer $i$

In the denominator of formula (48), two terms and three terms can be separately taken to obtain the approximate transfer functions of the first, second and third order systems. The system output corresponding to the step input can be obtained as follows:

$$
Y(s)=\frac{1}{s} \cdot G(s)
$$


The approximate first-order and second-order time-domain outputs of step input are obtained as follows:

$$
\begin{gathered}
y^{1}(t)=\frac{1}{\omega_{0}}-e^{-\frac{\omega_{0}}{\omega_{1}} t} \\
y^{2}(t)=\frac{1}{s_{1} \cdot s_{2}}+\frac{1}{s_{1}^{2}-s_{1} \cdot s_{2}} e^{s_{1} t}+\frac{1}{s_{2}^{2}-s_{1} \cdot s_{2}} e^{s_{2} t}
\end{gathered}
$$

Among them, $s_{1}$ and $s_{2}$ are the roots of the characteristic polynomial $\omega_{0}+$ $\omega_{1} s+\omega_{2} s^{2}=0$ of the second order transfer function. The calculation formulas are as follows:

$$
\begin{gathered}
s_{1}=\frac{\sqrt{-4 \omega_{2} \omega_{0}+\omega_{1}^{2}}-\omega_{1}}{2 \omega_{2}} \\
s_{2}=-\frac{\sqrt{-4 \omega_{2} \omega_{0}+\omega_{1}^{2}}+\omega_{1}}{2 \omega_{2}}
\end{gathered}
$$

\section{Model application}

Taking the heat transfer of high-temperature protective clothing which usually made of a multi-layered fabric material as an example. The protective clothing usually has four layers of composite layer, which are recorded as I, II, III and IV layers, and the first layer contacts with the external environment. There is also a gap between the third layer and the skin, which recorded as the forth layer. It is assumed that the fourth layer contacts with the skin is in an adiabatic state. The parameters of the composite [13] [14] are shown in Table 1.

Table 1. Various parameters of composite materials

\begin{tabular}{ccccc}
\hline Layer & $\begin{array}{c}\text { Density } \\
\left(\mathrm{kg} / \mathrm{m}^{3}\right)\end{array}$ & $\begin{array}{c}\text { Specific heat } \\
\left(\mathrm{J} /\left(\mathrm{kg} \cdot{ }^{\circ} \mathrm{C}\right)\right)\end{array}$ & $\begin{array}{c}\text { Thermal } \\
\text { conductivity } \\
\left(\mathrm{W} /\left(\mathrm{m} \cdot{ }^{\circ} \mathrm{C}\right)\right)\end{array}$ & $\begin{array}{c}\text { Thickness } \\
(\mathrm{mm})\end{array}$ \\
\hline I & 300 & 1377 & 0.082 & 0.6 \\
II & 862 & 2100 & 0.37 & 0.6 \\
III & 74.2 & 1726 & 0.045 & 3.6 \\
IV & 1.18 & 1005 & 0.028 & 0.5 \\
\hline
\end{tabular}

First of all, substituting the parameters above into the transfer function of the system function to compare the accuracy mathematical models in the second-order and third-order.

$$
G(s)=\frac{1}{1+199.4920 s+7.7728 \cdot 10^{3} \cdot s^{2}+1.2732 \cdot 10^{5} \cdot s^{3}+\cdots}
$$

The first three and the first four terms of the characteristic polynomial are obtained respectively, and the approximate second-order and third-order systems are obtained. The analytical solutions of the first-order second-order and third-order approximate step input responses of the heat transfer of the composite fabric layer are as follows: 


$$
\begin{gathered}
y_{1}(t)=1-e^{-0.0050 t} \\
y_{2}(t)=1+0.5690 e^{-0.0188 t}-1.5690 e^{-0.0068 t}
\end{gathered}
$$

$y_{3}(t)=0.9999-1.350 e^{-0.0065 t}+9.6093 \cdot 10^{-42} e^{-0.0273 t}[3.4159$. $\left.10^{39} \sin (0.0217 t)+3.5905 \cdot 10^{40} \cos (0.0217)\right]$

When calculating the heat transfer process of composite fabrics on Maple software platform, the time-domain responses of the first and second step are shown in Figure 2.The time-domain responses of the second and the third step are shown in and Figure 3. It can be seen that the difference between the first and second order responses is great, while the difference between the second and third order responses can be neglected. This can be proved by overlapping three curves in the same figure (Figure. 4). In order to make the calculation less complicated, it is enough to take the second-order model under the premise of accuracy allowance.

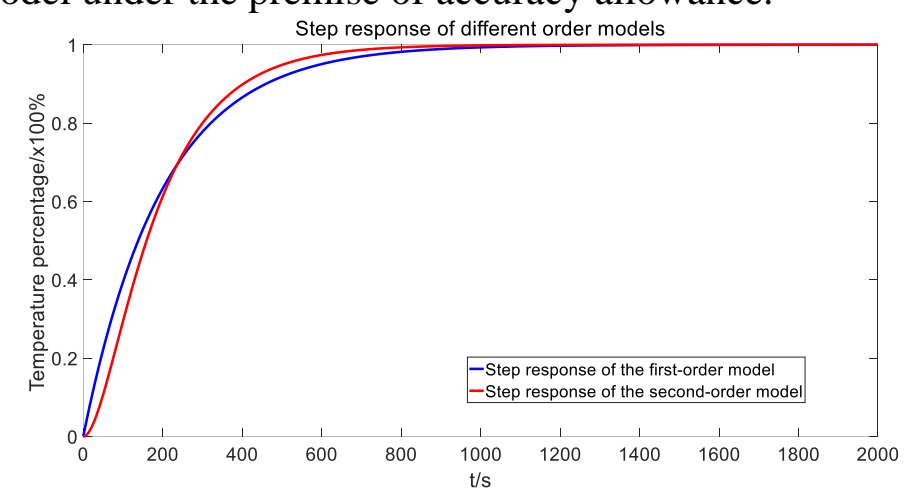

Figure. 2 Step response of $1 \& 2$ order model

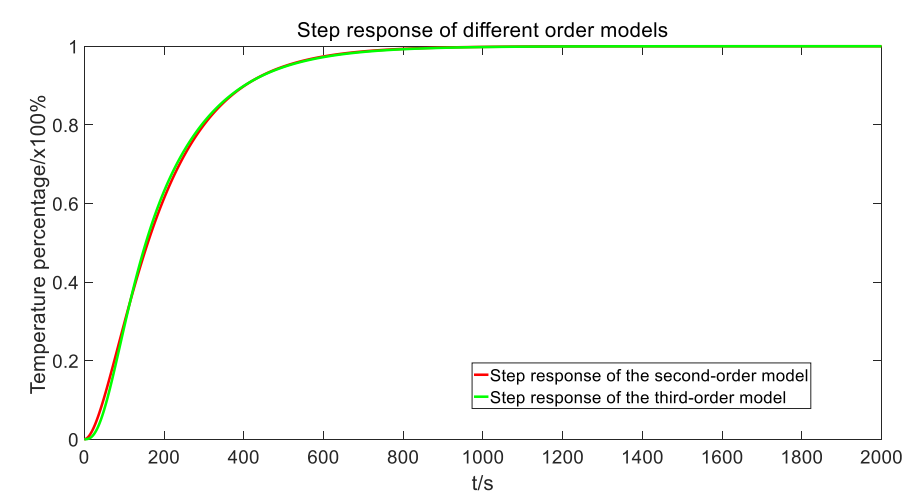

Figure. 3 Step response of the $2 \& 3$ order model

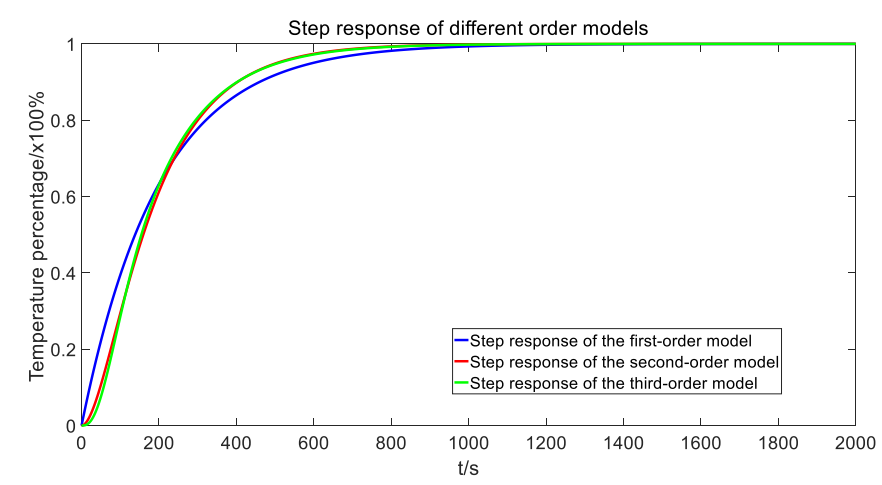

Figure. 4 Step response of 1-3 order model 


\section{Conclusion}

(1) Based on Fourier's law of heat transfer, the second-order approximate system function of heat transfer system is obtained by applying Laplace transform to the dynamic process of heat transfer of multilayer composites. T The error of the second-order system function can be neglected.

(2) The application of the formula proves that the dynamic heat transfer model of composite layer based on Laplace transform has high accuracy and can effectively reduce the experimental cost of temperature measurement.

(3) The dynamic heat transfer model of composite layer based on Laplace transform can be used for real-time on-line controlled, which has reference value for the study of heat transfer efficiency.

(4) In the case that we can only get the density, specific heat capacity, thickness, and thermal conductivity, the model can be used to analyze the heat transfer performance, which provides theoretical basis and analytical means for the optimization design and performance evaluation of composite materials.

\section{Reference}

[1] Li Defu, Yang Yuping, Liu Xiaoxu. Research status and prospects of heat transfer characteristics of multilayer insulation materials [J]. Spacecraft Environmental Engineering, 2013, 30 (03): 302-309.

[2] Wang Yicheng, Zhu Heyun. Chemical Process Control Engineering [M]. Beijing: Chemical Industry Press, 1991. 260-274.

[3] Chen Zhijiu, Yan Xiongcai, Ding Guoliang. Refrigeration system thermodynamics [M]. Second edition. Beijing: Mechanical Industry Press, 1998. 148-181.

[4] Study on heat transfer dynamic characteristics of Wang Zenggang composite sheet, [J].Journal of Hebei University of Science and Technology, 2006(01):10-14.

[5]Wang Zenggang, Liu Xiaoming, Li Wei. Approximation of heat transfer dynamics of composite plates[J]. Journal of Hebei University of Science and Technology, 2004(01): 5-8.

[6]Mahanthesh B.Gireesha, B.J.Gorla Rama Subba Reddy.Heat and mass transfer effects on the mixed convective flow of chemically reacting nanofluid past a moving/stationary vertical plate. [J].Alexandria Engeering Joural.2016,3,569-581. [7]Yang Minghan,Song Yong,Wang Jianye, Xu Peng,Zhang Guangyu. Temperature control characteristics analysis of lead-cooled fast reactor with natural circulation. [J]Annals of Nuclear Energy.2016,4,54-61

[8]Hussanan Abid,Salleh Mohd Zuki,Khan Ilyas,Shafie Sharidan. Convection heat transfer in micropolar nanofluids with oxide nanoparticles in water, kerosene and engine oil. [J] Journal of Molecular Liquids.2017,3,482-488

[9]Ai Zhiyong,Wang Lujun, Precise Solution to 3D Coupled Thermohydromechanical Problems of Layered Transversely Isotropic Saturated Porous Media. [J] International Journal of Geomechanics.2018,4,67-85

[10]H.J. Xu,Z.B. Xing,F.Q. Wang,Z.M. Cheng. Review on Heat Conduction, Heat Convection, Thermal Radiation and Phase Change Heat Transfer of Nanofluids in 
Porous Media: Fundamentals and Applications[J]. Chemical Engineering Science,2018.

[11]Hassan Eltayeb,Adem Kıliçman. A note on solutions of wave, Laplace's and heat equations with convolution terms by using a double Laplace transform[J]. Applied Mathematics Letters,2007,21(12).

[12] Yang Shiming, Tao Wenzhao, etc., Heat Transfer [M]. Beijing, Higher Education Press, 2006.8: 4 11, 45, 48 49.

[13]Chang Sheng, Li Jin. Effect of air layer on heat transfer performance of knitted fabrics[J]. Knitting Industry, 2015(07): 100-103.

[14] Chinese Society of Industrial and Applied Mathematics, 2018 Higher Education Society Cup National College Students Mathematical Modeling Competition Competition

http://www.mcm.edu.cn/html_cn/node/7cec7725b9a0ea07b4dfd175e8042c33.html, 2018-9-13 